\title{
La presión arterial y el tabaquismo son determinantes de evolución fatal tras un infarto agudo de miocardio
}

Preinfarction blood pressure and smoking are determinants for a fatal outcome of myocardial infarction. Njølstad I, Arnesen E. Arch Intern Med 1998;158:1326-1332.

Objetivo

Investigar factores de riesgo cardiovascular que puedan influenciar la sobrevida a corto plazo en pacientes que sufren un primer evento de infarto agudo de miocardio o muerte súbita.

\section{Diseño}

Estudio prospectivo, de cohortes, poblacional.

\section{Lugar}

Condado de Finmark, Noruega.

\section{Pacientes}

Se incluyeron inicialmente todos los pacientes de ambos sexos de 35 a 52 años residentes en el condado de Finmark durante 1974-5 y 1977-8 que respondieran un cuestionario enviado por correo (tasa de respuesta $90,5 \%$ ) y no tuvieran antecedentes de infarto de miocardio o accidente cerebrovascular. Esta población $(n=13315)$ fue seguida durante una media de 14 años, y se analizaron los 760 pacientes que sufrieron durante dicho período un primer evento de infarto agudo de miocardio o muerte súbita.

\section{Evaluación de factores pronósticos pectoris}

Variables determinadas al momento de inclusión al estudio: edad, tabaquismo, diabetes, angina tratamiento antihipertensivo, grado de actividad física, presión sistólica y diastólica, Indice de Masa Corporal, medición de colesterol total, HDL, triglicéridos y glucemia. Edad al momento del evento.

\section{Medición de resultados principales}

Mortalidad de cualquier causa ocurrida dentro de los 28 días posteriores a un primer infarto de miocardio o muerte súbita.

\section{Resultados Principales posteriores al evento}

La mortalidad del grupo total dentro de los 28 días del evento fue $30,5 \%$. La presión arterial sistólica al momento de inclusión al estudio fue la variable más fuertemente asociada con mortalidad a 28 días. La mortalidad fue del $24,1 \%$ en los pacientes con presión sistólica menor de $140 \mathrm{mmHg}$, de $34,0 \%$ entre 140 y $159 \mathrm{mmHg}$, y 46,8\% para el grupo con presión sistólica de $160 \mathrm{mmHg}$ o más.

En un análisis multivariable* , las variables que se asociaron independientemente con mayor mortalidad a 28 días fueron la presión sistólica y el tabaquismo en el momento de inclusión al estudio, y la edad al momento del evento. La angina de pecho y la diabetes tuvieron una fuerte tendencia pero sin significación estadística, probablemente por escaso número de pacientes dentro de estos subgrupos, mientras que otras variables no mostraron asociación con la mortalidad.

\begin{tabular}{lcc}
\hline Variable & Riesgo relativo & IC 95\% \\
\hline $\begin{array}{l}\text { Presión arterial sistólica } \\
\text { (por cada 15 mmHg) }\end{array}$ & 1,22 & $1,13-1,31$ \\
\hline Edad al evento (por cada 5 años) & 1,12 & $1,01-1,24$ \\
\hline Tabaquismo & 1,40 & $1,07-1,85$ \\
\hline Angina pectoris & 1,43 & $0,90-2,27$ \\
\hline Diabetes & 1,97 & $0,86-4,49$ \\
\hline
\end{tabular}

\section{Conclusiones}

En pacientes con un primer infarto agudo de miocardio o muerte súbita, el antecedente de presión arterial sistólica elevada y/o tabaquismo, y la edad al momento del infarto fueron predictores independientes de mortalidad a 28 días.

\section{COMENTARIO}

La enfermedad coronaria es la primera causa de muerte en el mundo occidental. Más de la mitad de dichas muertes son agudas y debidas a un "ataque cardíaco", englobando dentro de esta denominación a pacientes con infarto agudo de miocardio diagnosticado y a aquellos que sufren muerte súbita cardíaca, la que en general se debe también a un evento coronario agudo.

Desde el estudio Framingham en adelante, se han identificado distintos factores de riesgo asociados al infarto agudo de miocardio y la muerte súbita de origen cardíaco, siendo los principales la edad, el sexo, el perfll lipídico, el tabaquismo, la diabetes y la hipertensión arterial 1 .

Han sido realizados además varios estudios para determinar predictores pronósticos en pacientes con infarto agudo de miocardio ${ }^{2-4}$. Sin embargo, dichos estudios se efectuaron en general en poblaciones de pacientes con infarto agudo de miocardio en el hospital, lo que no da una verdadera idea de la magnitud del problema ya que no se incluyen los pacientes que no llegan al hospital por morir súbitamente.

El principal mérito de este estudio de Njølstad y Arnesen es incluir a todos los pacientes con un primer ataque cardíaco, con un enfoque po- blacional, lo que permite afirmar conclusiones que no se hubieran obtenido en estudios de otro tipo. Es especialmente importante la relación independiente detectada entre el tabaquismo y la mortalidad, que se contrapone a reportes previos que afirmaban que dicho antecedente es un predictor de mejor evolución post-infarto ${ }^{4}$. Visto en perspectiva, es dable suponer que lo que realmente ocurre es que los pacientes tabaquistas tienen mayor proporción de ataque cardíacos rápidamente fatales, y que la población de fumadores con infarto de miocardio que sobrevive y llega al hospital tiene mejor pronóstico porque es un grupo que ha sido seleccionado previamente.

En relación con la hipertensión sistólica, la relación con la mortalidad es clara e independiente de la edad y de otros predictores pronósticos. En resumen, una nueva prueba de la relación existente entre los factores de riesgo cardiovascular tradicionales y la morbimortalidad cardía$\mathrm{ca}$, y un nuevo mensaje para redoblar esfuerzos en la prevención de la cardiopatía isquémica.

*Ver Glosario

Dr. Carlos Boisonet

Servicio de Cardiología. Hospital Italiano de Buenos Aires.

Risferencias

Anderson K, Wilson P, Odell P, y col. An updated coronary risk profile. A statement for health professionals. Circulation 1991;83:356-362.

2. Gillum R, Folsom A, Luepker R, y col. Sudden death and acute myocardial infarction in a metropolitan area, 1970-1980: The Minnesota Heart Survey. N Engl J Med 1983;309:1353-1358.

2. Goldberg R. Gore J, Alpert J, y col. Incidence and case fatality rates of acute myocardial infarction (1975-1984): The Worcester Heart Attack Study. Am Heart J $1988 ; 115: 761-767$.

- Grines C, Topol E, O'Neill W, y col. Effect of cigarette smoking on outcome after thrombolytic therapy for myocardial infarction. Circulation 1995;91:298-303.

E. Lee KL, Woodlief LH, Topol EJ y col. Predictors of 30-day mortality in the era of reperfusion for acute myocardial infarction. GUSTO-I Investigators. Circulation, 1995; 91:6, 1659-1668. 